\title{
ECMO support for viral induced cardiogenic shock: a bridge too far?
}

\author{
Trevor J. Williams and Vincent Pellegrino \\ Affiliation: Alfred Hospital, Monash University, Melbourne Australia.
}

Correspondence: Trevor J. Williams, Respiratory Medicine, Alfred Hospital, Melbourne 3004, Australia. E-mail: t.williamsQalfred.org.au

@ERSpublications

Decision making in a COVID-19 pandemic may need to be undertaken in the absence of evidence from randomised controlled trials. A recent case study suggests VA-ECMO in severe COVID-19 sepsis and shock is highly unlikely to result in patient survival. https://bit.ly/2YjQPP3

Cite this article as: Williams TJ, Pellegrino V. ECMO support for viral induced cardiogenic shock: a bridge too far?. Eur Respir J 2020; 56: 2002129 [https://doi.org/10.1183/13993003.02129-2020].

The global coronavirus disease 2019 (COVID-19) pandemic has captured the world's conscious thought in a way not experienced in most of our lifetimes. In a world where Presidents conduct foreign policy by Twitter, we are not lacking information volume. In medicine, our version of the gold standard for truth is two well-conducted randomised controlled trials with concordant results. With the pandemic impacting health services so rapidly, decisions about treatment options may be required well ahead of this level of certainty.

In this issue of the European Respiratory Journal, SIEwEKE et al. [1] report their experience utilising veno-arterial (VA) extracorporeal membrane oxygenation (ECMO) and LV Impella unloading (so called ECMELLA) in influenza-induced myocarditis and shock. Despite such aggressive life support, all seven patients died, the outcome being significantly worse than propensity matched patients with cardiogenic shock either due to ischaemic heart disease or non-ischaemic cardiomyopathy. Clearly, the retrospective, observational nature of these findings are not definitive. Clinical trials of procedures like ECMO are extremely difficult to design, recruit to and typically have design flaws that mean they are not the definitive word. Furthermore, COVID-19 is not influenza. In times of a COVID-19 pandemic can the study by SIEWEKE et al. [1] inform decision-making at an individual or even system wide level?

ECMO as a rescue therapy for severe acute respiratory failure has been used for well over 50 years [2]. Early uncontrolled observational reports suggested clinical benefits. Two early randomised controlled trials showed no survival benefit $[3,4]$. In such an operator-dependent procedure, the negative result may have been attributable to the investigator skill, experience and disposition toward the comparator treatments. Indeed, ECMO use did not cease in adults with severe acute respiratory failure in response to these studies. Specialised centres continued to apply the therapy in selected patients. Technical improvements in ECMO technology (particularly heparin bonding of all exposed surfaces including the oxygenator) led to great optimism that the safety profile of ECMO had improved sufficiently to be a viable alternative to perseverance with mechanical ventilation. A greater understanding of ventilator-induced lung injury, coupled with much-improved ECMO technology, led to the notion that earlier implementation would be beneficial. This has seen many expert centres became more proactive in initiating ECMO, with observational studies noted improving outcomes. In an attempt to "definitively" resolve this issue, two further randomised controlled trials for severe acute respiratory failure were conducted by ECMO specialist centres. These studies reported likely improved survival $[5,6]$. However, trial design has likely 
robbed us of this highest level of evidence. In the first report, conventional ventilation was (mostly) performed at the referring (not ECMO) centre. Thus, patients had better survival if treated at an expert ECMO centre (even when a substantial number could continue conventional mechanical ventilation). In the second study, crossover to ECMO was permitted, rendering the sample size inadequate to reach the primary statistical endpoint. Unfortunately, with the challenges in designing and conducting such trials truly compelling evidence (level 1) may still not be available for the foreseeable future (if ever).

The 2009 H1N1 pandemic saw, for the first time, widespread application of ECMO for viral induced pneumonitis/acute respiratory distress syndrome in circumstances where the likelihood of death was high (or even regarded as inevitable). One case series reported a better than 70\% survival [7]. A subsequent meta-analysis reporting outcome of ECMO in the setting of influenza identified 13 appropriate studies from 2009 to 2014. Mortality ranged from 8-65\% [8] across the studies. None, however, had an appropriate conventional therapy arm. Thus, the real effect of veno-venous (VV)-ECMO on survival in respiratory failure is still unclear. Notwithstanding this, VV-ECMO utilisation in the setting of severe, potentially reversible respiratory failure, and even if irreversible as a bridge to lung transplantation has increased substantially from this time.

VA-ECMO for refractory cardiac failure presents even greater challenges in completing high quality clinical trials. This likely explains the absence of any prospective randomised trials despite much higher utilisation internationally than VV-ECMO. Trial evidence to justify the effectiveness of VA-ECMO for some specific forms of circulatory failure will emerge soon (led by ECMO for cardiac arrest trials) [9]. Some patients with severe cardiac failure due to biopsy-proven myocarditis are among the most rewarding cases to treat with VA-ECMO [10-12]. VA-ECMO as a salvage for severe heart failure, often allowing stabilisation, assessment, recovery or progression to left ventricular assist device and/or cardiac transplantation has become standard approach in many heart failure/transplant centres.

Sepsis causing refractory circulatory shock (unsupportable with inotropes or vasopressors) has highly variable reported outcomes [13-15]. By contrast, almost universal failure of VA-ECMO to prevent death is reported when used in patients with refractory septic shock [15]. Whether or not some forms of heart failure are reversible with VA-ECMO in septic shock is unclear. In total there are very few reported VA-ECMO survivors in the presence of severe septic shock.

We would speculate that those with primarily a cardiac aetiology (myocarditis) who develop features of sepsis might do well with VA-ECMO support. Patients, however, who present primarily with septic shock will have poor outcome despite VA-ECMO. How is this relevant to COVID-19?

SARS-CoV-2 (the virus causing COVID-19) is very contagious, in large part because most patients have mild or even no symptoms. Some, however, have a severe or prolonged illness due to pneumonitis and/or acute respiratory distress syndrome. These patients require hospitalisation for supportive therapies, including high flow oxygen, noninvasive ventilation (NIV), mechanical ventilation (including prone positioning) and ECMO. Despite progressive implementation of measures to support type 1 respiratory failure, early reports suggest that over half the patients requiring mechanical ventilation (including NIV) do not survive [16]. VV-ECMO support may be indicated in some of these patients [17]. Indeed, the US Food and Drug Administration has also recently approved ECMO support for COVID-19 patients [18]. More than 1000 ECMO runs for COVID-19 have been reported to the international Extracorporeal Life Support Organization (ELSO) registry with, to date, an estimated 50-55\% survival [19]. Many patients remain on VV-ECMO support and their ultimate outcome is unknown.

An early, COVID-19 hospitalised case series, from Wuhan, China reports 23\% of patients with cardiac injury [16]. Whether this represent primary myocardial pathology or is secondary to septic shock is unclear. In the ELSO registry, 9\% of ECMO is VA-ECMO. Whilst this may represent operator preference, in some the indication for VA-ECMO is likely, by the treating team, believed to be cardiogenic shock. COVID-19 presents us with patients with severe respiratory failure, as well as shock in the setting of sepsis and/or severe cardiac dysfunction. In this particular group of patients there appears to be a complete lack of literature to guide decision-making.

Thus, the report of SIEWEKE et al. [1] utilising VA-ECMO with Impella support for cardiogenic shock due to influenza in seven patients is important to consider. This very experienced group was unable to achieve any survivors utilising an advanced strategy of VA-ECMO with left ventricular support via an impeller device. Propensity-matched patients with cardiogenic shock due to ischaemic heart disease or non-ischaemic cardiomyopathy fared better and support the clinical impression that refractory sepsis-related circulatory failure is lethal. The reporting of a "negative" study by a high impact journal is notable in itself as indicative of the need for information to guide contemporary practice. The report is clearly not the last word in treatment of myocarditis due to COVID-19. The numbers are small, two had 
out of hospital cardiac arrests, another two in hospital cardiac arrest. The propensity matching with such small numbers and the severity scoring systems cannot substitute for a proper, large randomised controlled trial.

What is apparent is that when we are discussing intensive care therapies for severe COVID-19 with patients and their families, a potentially long and arduous time in the intensive care unit is to be expected. Regrettably, the risk is substantial that the patient will not survive despite best efforts (including advanced therapies). It seems that in patients with severe respiratory failure, the already available outcome data suggest the use of VV-ECMO will save lives (as seen in the $2009 \mathrm{H} 1 \mathrm{~N} 1$ pandemic). What the report of SIEWEKE et al. [1] reflects is that, when severe shock develops in the setting of cardiac dysfunction, the use of VA-ECMO with or without additional left ventricular support appears highly unlikely to be lifesaving. Thus, in COVID-19 patients with sepsis and heart failure, avoid undue optimism in conversations with patients and next of kin. For healthcare systems, facing enormous demands for resources, VA-ECMO for such patients may reasonably regarded as inappropriate: a bridge too far.

Conflict of interest: None declared.

\section{References}

1 Sieweke J-T, Akin M, Stetskamp S, et al. Mechanical circulatory support in refractory cardiogenic shock due to influenza virus-related myocarditis. Eur Respir J 2020; 56: 2000925.

2 Gille JP, Bagniewski AM. Ten years of use of extracorporeal membrane oxygenation (ECMO) in the treatment of acute respiratory insufficiency (ARI). Trans Am Soc Artif Intern Organs 1976; 22: 102-109.

3 Zapol WM, Snider MT, Hill JD, et al. Extracorporeal membrane oxygenation in severe acute respiratory failure. A randomized prospective study. JAMA 1979; 242: 2193-2196.

4 Morris AH, Wallace CJ, Menlove RL, et al. Randomized clinical trial of pressure-controlled inverse ratio ventilation and extracorporeal $\mathrm{CO}_{2}$ removal for adult respiratory distress syndrome. Am J Respir Crit Care Med 1994; 149: 295-305.

5 Peek GJ, Mugford M, Tiruvoipati R, et al. Efficacy and economic assessment of conventional ventilatory support versus extracorporeal membrane oxygenation for severe adult respiratory failure (CESAR): a multicentre randomised controlled trial. Lancet 2009; 374: 1351-1363.

6 Combes A, Hajage D, Capellier G, et al. Extracorporeal membrane oxygenation for severe acute respiratory distress syndrome. N Engl J Med 2018; 378: 1965-1975.

7 Australia and New Zealand Extracorporeal Membrane Oxygenation Influenza Investigators, Davies A, Jones D, et al. Extracorporeal Membrane Oxygenation for 2009 Influenza A(H1N1) Acute Respiratory Distress Syndrome. JAMA 2009; 302: 1888-1895.

8 Sukhal S, Sethi J, Ganesh M, et al. Extracorporeal membrane oxygenation in severe influenza infection with respiratory failure: a systematic review and meta-analysis. Ann Card Anaesth 2017; 20: 14-21.

9 Belohlavek J, Kucera K, Jarkovsky J, et al. Hyperinvasive approach to out-of hospital cardiac arrest using mechanical chest compression device, prehospital intraarrest cooling, extracorporeal life support and early invasive assessment compared to standard of care. A randomized parallel groups comparative study proposal. 'Prague OHCA study'. J Transl Med 2012; 10: 163.

10 Pozzi M, Banfi C, Grinberg D, et al. Veno-arterial extracorporeal membrane oxygenation for cardiogenic shock due to myocarditis in adult patients. J Thorac Dis 2016; 8: E495-E502.

11 Matsumoto M, Asaumi Y, Nakamura Y, et al. Clinical determinants of successful weaning from extracorporeal membrane oxygenation in patients with fulminant myocarditis. ESC Heart Fail 2018; 5: 675-684.

12 Mirabel M, Luyt CE, Leprince P, et al. Outcomes, long-term quality of life, and psychologic assessment of fulminant myocarditis patients rescued by mechanical circulatory support. Crit Care Med 2011; 39: 1029-1035.

13 Brechot N, Luyt CE, Schmidt M, et al. Venoarterial extracorporeal membrane oxygenation support for refractory cardiovascular dysfunction during severe bacterial septic shock. Crit Care Med 2013; 41: 1616-1626.

14 Falk L, Hultman J, Broman LM. Extracorporeal membrane oxygenation for septic shock. Crit Care Med 2019; 47: 1097-1105.

15 Ro SK, Kim WK, Lim JY, et al. Extracorporeal life support for adults with refractory septic shock. J Thorac Cardiovasc Surg 2018; 156: 1104-1109.

16 Yang X, Yu Y, Xu J, et al. Clinical course and outcomes of critically ill patients with SARS-CoV-2 pneumonia in Wuhan, China: a single-centered, retrospective, observational study. Lancet Respir Med 2020; 8: 475-481.

17 MacLaren G, Fisher D, Brodie D. Preparing for the most critically ill patients with COVID-19: the potential role of extracorporeal membrane oxygenation. JAMA 2020; in press [https://doi.org/10.1001/jama.2020.2342].

18 US Food and Drug Administration. Coronavirus (COVID-19) Update: Daily Roundup April 7, 2020. www.fda.gov/ news-events/press-announcements/coronavirus-covid-19-update-daily-roundup-april-7-2020 Date last updated: 7 April 2020.

19 Extracorporeal Life Support Organization. ECMO in Corona Pandemics. www.euroelso.net/covid-19 